\title{
Generalized Parton Distributions at CLAS
}

\author{
Silvia Pisano (for the CLAS Collaboration) \\ Institut de Physique Nucléaire d'Orsay 91405 Orsay, FRANCE
}

\begin{abstract}
Generalized Parton Distributions represent one of the most exhaustive tools to describe nucleon structure. By combining together information from the electromagnetic form factors and from the standard parton distributions, they provide a three-dimensional picture of hadrons. The experimental access to these quantities is provided by the Deeply Virtual Compton Scattering and the Deeply Virtual Meson Production processes. The specific characteristics of the CEBAF Large Acceptance Spectrometer installed at the Thomas Jefferson National Accelerator Facility, with its high capability to reconstruct the final states of these exclusive processes over a large phase space, make this detector one of the best tool for the experimental investigation of the GPDs.
\end{abstract}

\section{Introduction}

For the past decades, a large amount of theoretical and experimental activity has been devoted to the understanding of hadron structure, but a full comprehension of mesons and baryons in terms of QCD degrees of freedom, namely quarks and gluons, is still lacking. Quantities as the hadron mass or spin, for example, are not yet fully described in terms of the properties of the elementary constituents, as the recent "spin crisis" proved.

These open questions call, then, for a more detailed investigation of hadron structure. To this end, new quantities have been introduced about ten years ago. These are the Generalized Parton Distributions (GPD) [1,2]. GPDs are defined as matrix elements of quark and gluon operators at a light-like separation (i.e. for a null light-cone time, $x^{+}=x^{0}+x^{3}=0$ ) $[3,4]$. These functions depend on three variables: $x$, the quark longitudinal momentum fraction, $\xi$, the longitudinal fraction of the four-momentum transfer, and $t$, the squared four-momentum transfer to the target. At the twist-two level, there are two spinindependent and two spin-dependent GPDs for each quark flavour, namely $\mathrm{E}(\mathrm{x}, \xi, \mathrm{t}), \mathrm{H}(\mathrm{x}, \xi, \mathrm{t})$ and $\tilde{\mathrm{E}}(\mathrm{x}, \xi$, $\mathrm{t}), \tilde{\mathrm{H}}(\mathrm{x}, \xi, \mathrm{t})$. The first moments in $x$ of the GPDs link them to the electromagnetic form factors, while at $t=0, \xi=0 \mathrm{H}(\mathrm{x}, \xi=0, \mathrm{t}=0)$ and $\tilde{\mathrm{H}}(\mathrm{x}, \xi=0, \mathrm{t}=0)$ reduce to the quark longitudinal momentum and helicity distributions, respectively $q(x)$ and $\Delta q(x)$.

From an experimental point of view, one can access these functions through two main processes: the Deeply Virtual Compton Scattering (DVCS) and the Deeply Virtual Meson Production (DVMP) (Fig. 1). These two processes are based on the so-called "handbag" mechanism (cfr. Fig. 1). Through the use of factorization theorems $[1,2,6]$, it has been proven both for the DVCS (for transverse photons) and for the DVMP (for longitudinal photons) that the process can be separated in a hard scattering part, well described through the tools of Quantum ElectroDynamics and/or Quantum CromoDynamics, and a non-perturbative part, that encodes the complex strong dynamics governing the existence of the hadron bound states and that is described with the GPDs.

Depending on the polarization degrees of freedom acting in the process (like, for example, the simultaneous presence of polarization in the beam and in the target, or the use of a polarized beam with an unpolarized target), various combinations of GPDs can be investigated.

Factorization is expected to hold for the DVCS (ep $\rightarrow e^{\prime} p^{\prime} \gamma$ ) at relatively low photon virtuality, i.e. in a range that can be explored at Jefferson Lab. However at these energies, an additional mechanism that gives a significant contribution to the $e p \gamma$ final state is the Bethe-Heitler process, where the final photon is emitted by the incoming or outgoing photon, and not by the interacting quark. Consequently, the 
amplitude for the process $e p \rightarrow e^{\prime} p^{\prime} \gamma$ is the sum of two contributions, namely:

$$
T_{e p \rightarrow e^{\prime} p^{\prime} \gamma}=T^{B H}+T^{D V C S},
$$

that give rise to an interference term at the cross-section level. In order to extract information on the GPDs, the cross section is decomposed in spherical harmonics, where the three contributions - DVCS, $\mathrm{BH}$ and the interference term (INT) - are isolated. Furthermore, the following asymmetry is introduced:

$$
A=\frac{d^{4} \vec{\sigma}-d^{4} \overleftarrow{\sigma}}{d^{4} \vec{\sigma}+d^{4} \overleftarrow{\sigma}}
$$

where the arrows correspond to beam helicities +1 and -1 . The BH importance is turned into an advantage for the GPD extraction by means of the interference term occurring in the cross section of the process $e p \rightarrow e^{\prime} p^{\prime} \gamma$, that survives in the asymmetry. INT, indeed, is amplified by the presence of the Bethe-Heitler part, whose contribution is well calculated in the framework of QED and can thus be disentangled from the one of the DVCS, where GPD appears. The numerator of the asymmetry turns out to be related to the GPDs through

$$
d^{4} \vec{\sigma}-d^{4} \overleftarrow{\sigma} \sim \sin \phi\left[F_{1} H(\xi, \xi, t)+k_{1}\left(F_{1}+F_{2}\right) \tilde{H}(\xi, \xi, t)+k_{2} F_{2} E(\xi, \xi, t)\right]
$$

where $F_{1}, F_{2}$ are the Dirac and Pauli form factors of the nucleon, $k_{1}, k_{2}$ are kinematical quantities, and $\phi$ is the angle between the $\gamma^{*} \gamma$ plane and the electron scattering plane [5]. At the leading twist, the asymmetry can then be written as

$$
A=\frac{a \sin \phi}{1+c \cos \phi+d \cos 2 \phi},
$$

where the parameters $a, c$ and $d$ can be expressed in terms of harmonic coefficients for DVCS, BH and INT. In particular, the coefficients for DVCS and INT can be expressed in terms of GPD integrals, the so-called Compton Form Factors. In the case of the GPD $H$, for instance, $\mathcal{H}$ is defined through

$$
\begin{aligned}
\Re e \mathcal{H} & =\mathcal{P} \int_{-1}^{1} \mathrm{~d} x\left[\frac{2 x}{\xi^{2}-x^{2}}\right] H(x, \xi, t), \\
\frac{1}{\pi} \Im m \mathcal{H} & =H(\xi, \xi, t)-H(-\xi, \xi, t) .
\end{aligned}
$$

A measurement of the asymmetry of Eq. 4 constitutes, then, a first step toward the extraction of the GPDs.

Deeply Virtual Meson Production represents another strategic tool for the comprehension of hadron structure since, as well as DVCS, it allows to access information on GPDs. By measuring different channels one can operate a flavour-decomposition that gives access to the GPDs of each single quark flavour. Among the various channels experimentally accessible, the process $e p \rightarrow e^{\prime} p \rho^{0}$, that brings to the production on the final state of a $\rho^{0}$ meson, is one of the most exstensively investigated, since predictions of its cross-sections are available both in terms of models based on the hadronic degrees of freedom, like those that make use of Regge theory [7-14], and in terms of GPD models, that describe the hadron dynamics in terms of partonic degrees of freedom [1-4, 6, 15, 16].

Among the hadronic models, the one tested through the CLAS data is the so-called JML model [7-10]. It is based on the Regge approach, according to which the reaction mechanism at the base of the meson electroproduction is the exchange of meson "trajectories" in the $t$-channel (Fig. 2). In the case of the $\gamma^{*} p \rightarrow p \rho^{0}$ channel the main contribution comes from the exchange of the $f_{2}(1270)$ and $\sigma$ mesons.

On the other hand, as far as the GPD-based models are concerned, their validity regime begins as soon as the Bjorken region is reached. An important topic is to understand what is the $Q^{2}$ value for which the validity of such a treatment starts. It will give information on the transition between the energy regime 

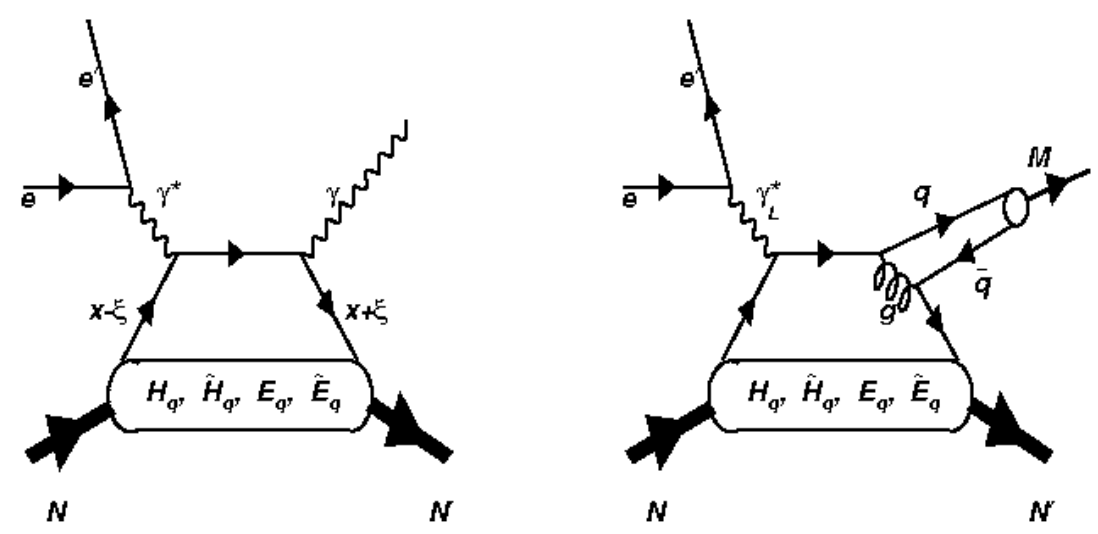

Fig. 1: Handbag diagram mechanism and factorization for both DVCS (left) and DVMP (right).

in which the hadron can be treated in terms of hadronic degrees of freedom and the one where a more elaborated, elementary "quantum field theory"-like approach starts to be needed. It is important to stress that for mesons the factorization has been proved only for the longitudinal part of the cross section, and consequently the experimental data analysis has to separate the longitudinal and transverse parts of the cross sections.
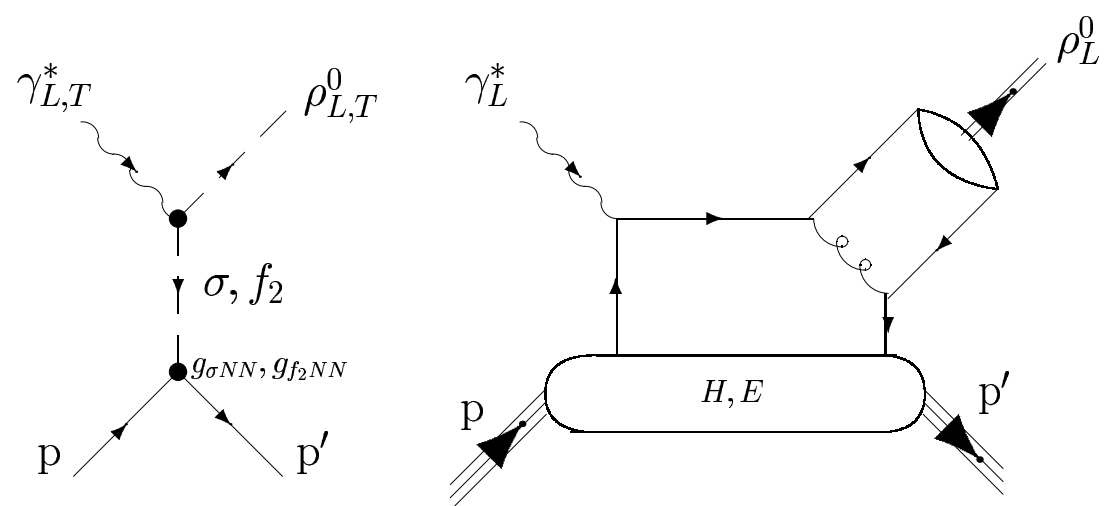

Fig. 2: The mechanisms for $\rho^{0}$ electroproduction at JLab energies for low $Q^{2}$ (left) through the exchange of mesons and for high $Q^{2}$ (right) through the quark exchange "handbag" mechanism (valid for longitudinal photons) where $H$ and $E$ are the unpolarized GPDs.

\section{Hall-B e1-dves experiment}

The experiment aiming to the extraction of the DVCS beam-spin asymmetry (BSA) took place in the Hall-B at the Thomas Jefferson National Accelerator Facility. The Continuous Electron Beam Accelerator Facility (CEBAF) offers a unique environment for hadronic physics measurements, providing a continuous electron beam with energies up to $6 \mathrm{GeV}$. The e1-dvcs experiment used the CEBAF 5.77$\mathrm{GeV}$ electron beam with an average polarization $P=0.794$, a 2.5 -cm-long liquid-hydrogen target and the CEBAF Large Acceptance Spectrometer (CLAS) [17]. In order to effectively reconstruct all the three final-state particles from the reaction $e p \rightarrow e p \gamma$ (and in particular the 1-to-5 GeV DVCS photons emitted between $4.5^{\circ}$ and $15^{\circ}$ with respect to the beam direction), a new inner calorimeter (IC) was added to the standard CLAS configuration, $55 \mathrm{~cm}$ downstream from the target. The operating luminosity was $2 \times 10^{34} \mathrm{~cm}^{-2} \mathrm{~s}^{-1}$. 

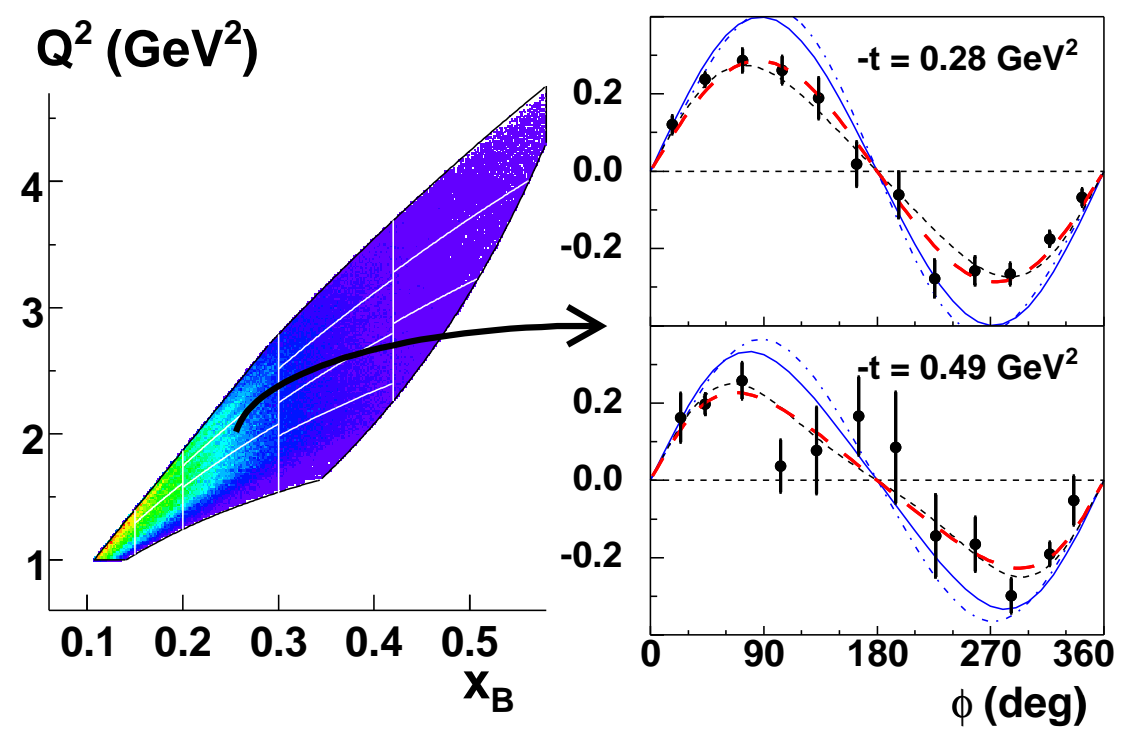

Fig. 3: Left (from Ref. [18]): kinematic coverage and binning in the $\left(x_{B}, Q^{2}\right)$ space. Right (from Ref. [18]): $A(\phi)$ for 2 of the $62\left(x_{B}, Q^{2}, t\right)$ bins, corresponding to $\left\langle x_{B}\right\rangle=0.249,\left\langle Q^{2}\right\rangle=1.95 \mathrm{GeV}^{2}$, and two values of $\langle t\rangle$. The red long-dashed curves correspond to fits with Eq. (4) (with $d=0$ ). The black dashed curves correspond to a Regge calculation [19]. The blue curves correspond to the GPD calculation described in the text, at twist-2 (solid) and twist-3 (dot-dashed) levels, with $H$ contribution only.

Exclusive ep $\gamma$ events were selected by detecting the 3 particles in CLAS and removing the background via the application of exlcusivity cuts [18]. The data were divided into thirteen bins in the $\left(x_{B}, Q^{2}\right)$ space as per Fig. 3, five bins in $-t$ (defined by the bin limits $0.09,0.2,0.4,0.6,1$ and $1.8 \mathrm{GeV}^{2}$ ) and twelve $30^{\circ}$-wide bins in $\phi$. BSA were extracted for each $\left(x_{B}, Q^{2}, \phi, t\right)$ bin. The parameter $d$ in the denominator of Eq. 4 is expected to be smaller than 0.05 over our kinematic range, and indeed it was found to be compatible with zero, within statistical accuracy, when included in the fit. Two examples of the measured asymmetry are reported in Fig. 3, while in Fig. $4 a=A\left(90^{\circ}\right)$ as a function of $-t$ is shown. Together with the experimental data two fits are shown, one corresponding to the hadronic model proposed in Ref. [19], and the other corresponding to the GPD calculation in Ref. [14].

As to the GPD interpretation, constrained parametrizations have been developed starting from the various models, in order to test their phenomenological effectiveness. In Ref. [14] the following parametrization is proposed:

$$
\begin{aligned}
& H=\sum_{q} e_{q}^{2}\left\{\int_{-1}^{+1} \mathrm{~d} \beta \int_{-1+|\beta|}^{1-|\beta|} \mathrm{d} \alpha \delta(x-\beta-\xi \alpha) \mathfrak{h}^{q}(\beta, \alpha, t)\right. \\
&+\left.\theta\left(1-\frac{x^{2}}{\xi^{2}}\right) D^{q}\left(\frac{x}{\xi}, t\right)\right\} \\
& \text { with } \quad \mathfrak{h}^{q}(\beta, \alpha, t)=q(\beta) \pi_{b}(\beta, \alpha) e^{-\alpha_{1}^{\prime}(1-\beta) t},
\end{aligned}
$$

where $e_{q}$ and $q(\beta)$ are the electric charge and unpolarized parton distribution for quark flavor $q, \pi_{b}$ a profile function [20] and $\alpha_{1}^{\prime}$ is a Regge slope adjusted to recover the proton form factor $F_{1}$ from the first moment of the GPD $H$. Eq. (8) extends the ansatz of Ref. [14] for the $t$ dependence to non-zero values of $\xi$. The $D$ term in Eq. (7) is calculated within a quark-soliton chiral model [16]. Using predetermined parameters, the calculations of beam-spin asymmetries yield the solid and dot-dashed curves in Figs. 3 and 4 , without and with a twist-3 term calculated in the Wandzura-Wilczek approximation [16]. The 


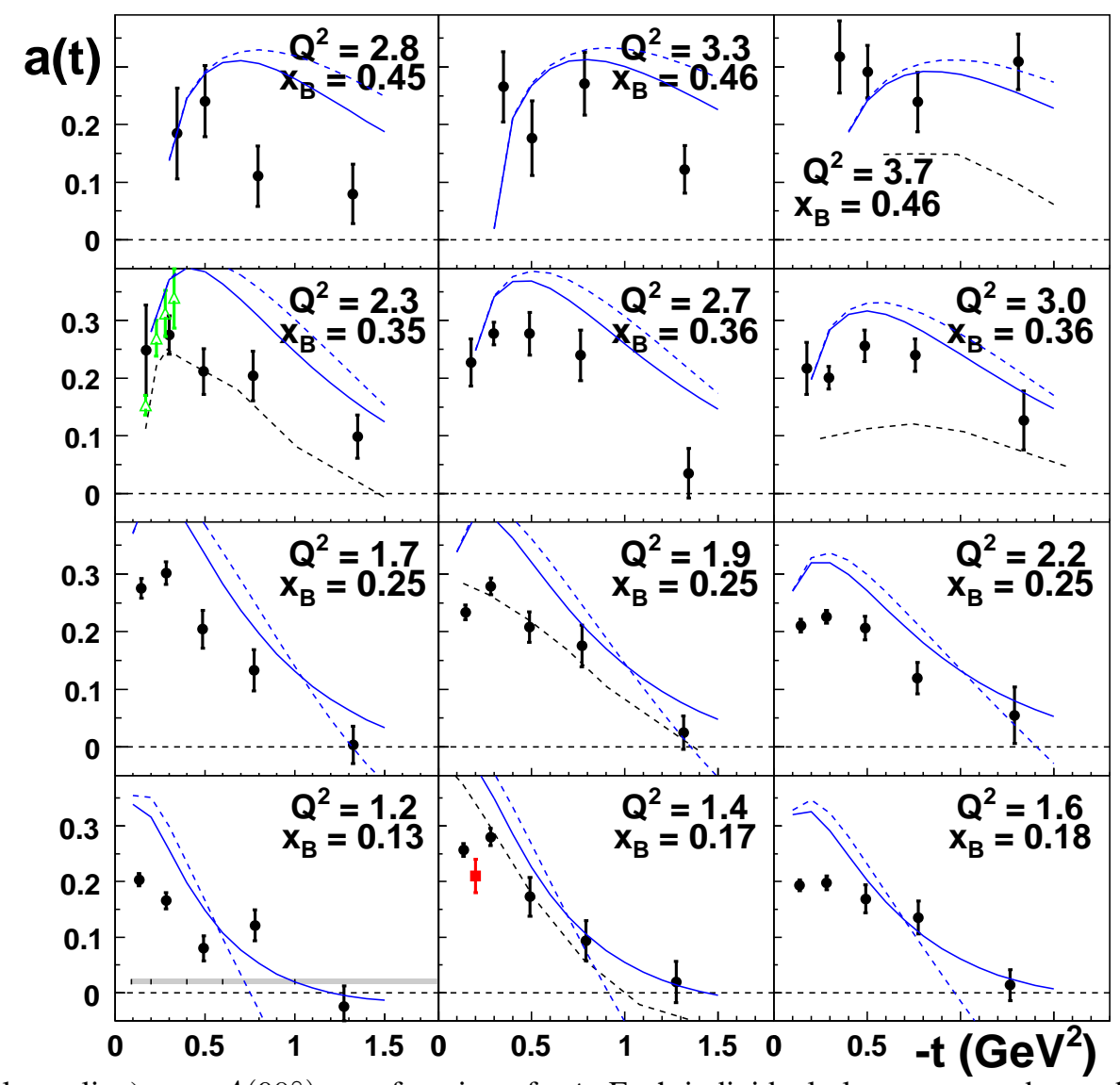

Fig. 4: (Color online) $a=A\left(90^{\circ}\right)$ as a function of $-t$. Each individual plot corresponds to a bin in $\left(x_{B}, Q^{2}\right)$. Systematic uncertainties and bin limits are illustrated by the grey band in the lower left plot. Black circles are from Ref. [18]. Previous CLAS results are from Ref. [21] (red square) or extracted from cross section measurements [22] (green triangles), at similar - but not equal - values of $\left\langle x_{B}\right\rangle$ and $\left\langle Q^{2}\right\rangle$. See Fig. 3 caption for the description of the curves.

predictions overestimate the measured asymmetries at low $|t|$, especially for small values of $x_{B}$ and/or $Q^{2}$.

\section{Hall-B e1-6 experiment}

In this section, we refer to the results presented by the CLAS Collaboration in Ref. [23]. The data for the measurement of the $e p \rightarrow e^{\prime} p \rho^{0}$ cross-section were taken with an electron beam having an energy of $5.754 \mathrm{GeV}$ impinging on an unpolarized 5-cm-long liquid-hydrogen target. The integrated luminosity of the data set was $28.5 \mathrm{fb}^{-1}$, and its kimematic domain corresponds approximately to $Q^{2}$ from 1.5 to $5.5 \mathrm{GeV}^{2}$. The analyzed data have a $W$, the $\gamma^{*}-p$ center-of-mass energy, greater than $1.8 \mathrm{GeV}$, which corresponds to a range of $x_{B}$ approximatively from 0.15 to 0.7 . As mentioned before, since the factorization theorems for the meson electroproduction have been proven only for the longitudinal component of the cross-section, a trasverse-longitudinal separation is needed for $\sigma_{\gamma^{*} p \rightarrow p \rho^{0}}$. Such a separation is realized by analyzing the decay-pion angular distribution in the $\rho^{0}$ center-of-mass frame and relying on the $s$-channel helicity conservation.

The results for the cross-sections are reported in Figs. 5 and 6. In particular, Fig. 5 shows the results for the reduced cross sections $\gamma_{L}^{*} p \rightarrow p \rho_{L}^{0}$ as a function of $W$ for constant $Q^{2}$ bins, in units of $\mu \mathrm{b}$, together with the world data. The superimposed curves represent fits obtained with the Regge JML calculation 


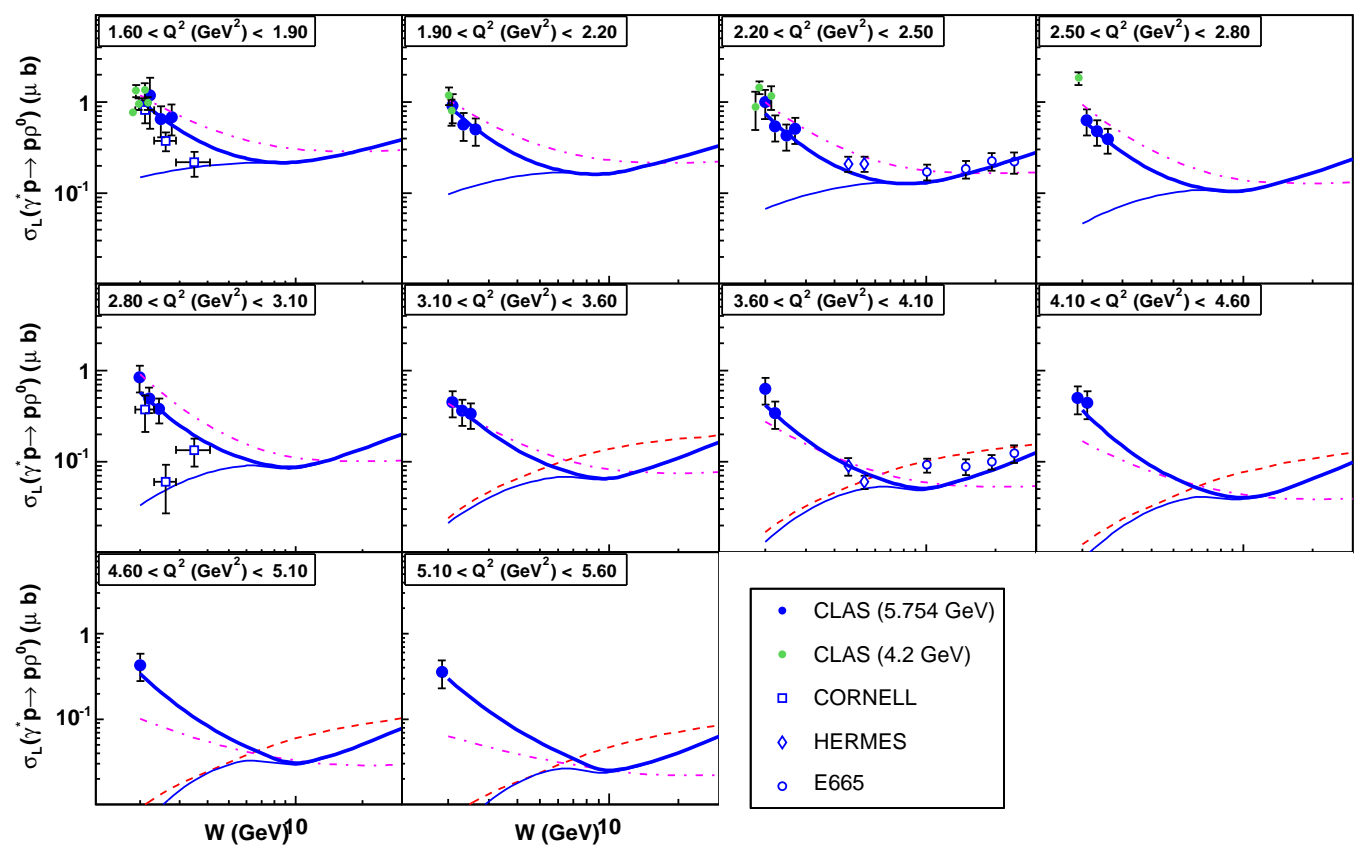

Fig. 5: World data for the reduced cross sections $\gamma_{L}^{*} p \rightarrow p \rho_{L}^{0}$ as a function of $W$ for constant $Q^{2}$ bins, in units of $\mu \mathrm{b}$. The dashed curves show the results of the GK calculation and the thin solid curves show the results of the VGG calculation. Both calculations are based on Double Distributions as proposed in Ref. [24] for the GPD parametrizations and incorporate higher twist effects through $k_{\perp}$ dependence. The thick solid curve is the VGG calculation with the addition of a D-term inspired contribution [11-14]. The dot-dashed curve shows the results of the Regge JML calculation. The 4.2 GeV CLAS, CORNELL, HERMES and E665 data are respectively from Refs. [25], [26], [27] and [28].

[7-10] and with GPD-inspired models, in particular the VGG [11-14] and the GK [16] ones. These two models parametrize the $(x, \xi)$ dependence of the $H$ and $E$ GPDs with double distributions, following the prescription in Ref. [24], while they differ in the treatment of the $t$ dependence and in the way they sum up the two handbag contributions (namely the one due to the quark GPD and the one coming from the gluon part). In fact, the GK group sums the two handbag diagrams at the amplitude level, while the VGG group has treated them at the cross section level neglecting, in this way, the interference between them. As it is clearly visible in Fig. 5, two different behaviours emerge for the cross-section: at low $W$, indeed, $\sigma_{L}$ decreases with $W$, while it starts to rise again at $W \approx 10 \mathrm{GeV}$. The JML model (dash-dotted line) reproduces fairly well the two general behaviors just mentioned, but it drops as a function of $Q^{2}$ faster than the data and agrees with them only up to $Q^{2} \approx 4.10 \mathrm{GeV}^{2}$. As to the GPD approaches (see caption of Fig. 5 for the legend), they give a good description of the high and intermediate $W$ region, down to $W \approx 5 \mathrm{GeV}$. At high $W$ the slow rise of the cross section is due to the gluon and sea contributions, while the valence quarks contribute only at small $W$. Since the VGG model misses the interference term - because it sums up the two contributions at the cross-section level - it differs the most from the GK model at intermediate $W$, where the inference is maximal, since it corresponds to the regime where the gluon contribution starts to play a role in the process, while the quark one is still significant. Both the GPD-based models fail to reproduce the low- $W$ region. This occurrence can have a two-fold origin. On the one hand, it can be due to a failure of the handbag description (whose validity regime for DVMP is not completely understood yet) at the low- $W$ region. On the other hand, another source of complexity can be indentified in the presence of a second, non-perturbative element in the integrals defining the GPDs 
in the VGG and GK models, i.e. the meson amplitude. This second contribution is indeed convoluted with the forward distribution $q(x)$, that in principle defines the $x$-dependence of the GPDs. Fig. 6 shows the longitudinal differential cross section $d \sigma_{L} / d t$ as a function of $t$. The dash-dotted curves show the JML model, where $t$-dependent form factors have been introduced at the electromagnetic vertices of the diagrams for the meson exchange [7] in order to enrich the "natural" $t$ dependence given by the Regge formula $s^{\alpha(t)}$.

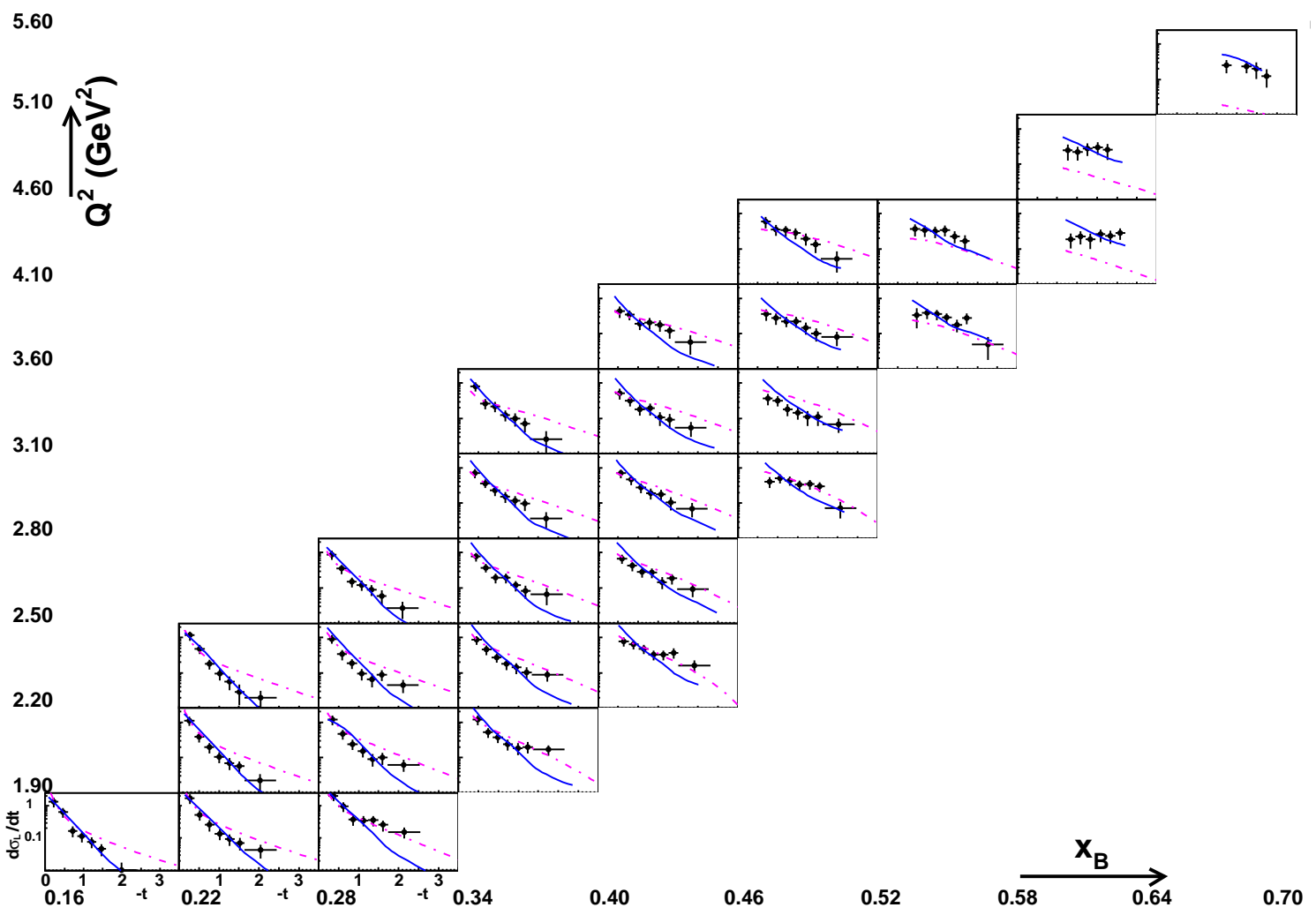

Fig. 6: Longitudinal cross sections $d \sigma_{L} / d t$ (in $\mu \mathrm{b} / \mathrm{GeV}^{2}$ ) for all bins in $\left(Q^{2}, x_{B}\right)$ as a function of $t$ (in $\mathrm{GeV}^{2}$ ). The thick solid curve represents the results of the VGG calculation with the addition of a generalized D-term [11-14]. The dash-dotted curves are the results of the JML model calculation.

\section{Conclusions}

GPDs are a powerful tool for the description of hadron properties, since they are able to provide a threedimensional picture of their structure starting from the quark and gluon fields. It addresses, in this way, the strategic question of how the basic fields of the QCD Lagrangian, namely quark and gluon fields, are related to the observed degrees of freedom, $i$. e. the hadrons. GPDs are experimentally accessible through deeply exclusive processes like DVCS and DVMP. The CLAS detector at the CEBAF facility is very suitable for the measurement of these reactions, as the data set shown above prove. As to the actual extraction of the GPDs, data from both the DVCS and DVMP process should properly be combined, since the two processes turn out to be sensible to different GPDs and can give, in this way, complementary information on them.

As to the data presently available, results from the CLAS e1-dvcs experiment - that produced the most extensive set of DVCS data up to date - show that the beam-spin asymmetries exhibit the expected sinusoidal dependence on $\phi$. Furthermore, the presently existing models are being tested on the CLAS data. As to DVMP, CLAS provided measurements for the cross sections of the process $e p \rightarrow e^{\prime} p \rho^{0}$, that will allow to put further constraints on the presently available GPD-based models and that will shed light 
on the interplay between descriptions of the nucleon structure in terms of hadronic or partonic degrees of freedom.

\section{References}

[1] X. Ji, Phys. Rev. Lett. 78 (1997) 610; Phys. Rev. D 55 (1997) 7114.

[2] J.C. Collins, L. Frankfurt and M. Strikman, Phys. Rev. D 56 (1997) 2982.

[3] M. Diehl, Phys. Rept. 388 (2003) 41.

[4] A.V. Belitsky and A.V. Radyushkin, Phys. Rept. 418 (2005) 1.

[5] A.V. Belitsky and A.V. Radyushkin, Phys. Rept. 418 (2005) 1.

[6] D. Müller, D. Robaschik, B. Geyer, F.-M. Dittes, and J. Horejsi, Fortschr. Phys. 42 (1994) 101.

[7] J. M. Laget, Phys. Rev. D 70 (2004) 054023.

[8] J.-M. Laget, Phys. Lett. B 489 (2000) 313.

[9] F. Cano and J.-M. Laget, Phys. Rev. D 65 (2002) 074022.

[10] F. Cano and J. M. Laget, Phys. Lett. B 551 (2003) 317.

[11] M. Vanderhaeghen, P.A.M. Guichon, and M. Guidal, Phys. Rev. Lett. 80 (1998) 5064.

[12] M. Vanderhaeghen, P.A.M. Guichon, and M. Guidal, Phys. Rev. D 60 (1999) 094017.

[13] K. Goeke, M.V. Polyakov and M. Vanderhaeghen, Prog. Part. Nucl. Phys. 47 (2001) 401.

[14] M. Guidal, M.V. Polyakov, A.V. Radyushkin and M. Vanderhaeghen, Phys. Rev. D72 (2005) 054013.

[15] A.V. Radyushkin, Phys. Lett. B 380 (1996) 417; Phys. Rev. D 56 (1997) 5524.

[16] K. Goeke, M. V. Polyakov and M. Vanderhaeghen, Prog. Part. Nucl. Phys. 47 (2001) 401.

[17] B. A. Mecking et al. Nucl. Instr. Meth. A503 (2003) 513.

[18] F.X. Girod, Phys.Rev.Lett. 100 (2008) 162002.

[19] J. M. Laget, Phys. Rev. C 76 (2007) 052201.

[20] A.V. Radyushkin, in At the Frontier of Particle Physics - Handbook of QCD, M. Shifman Ed. (World Scientific, Singapore 2001), p. 1037.

[21] S. Stepanyan et al. Phys. Rev. Lett. 87 (2001) 182002.

[22] C. Muñoz Camacho et al., Phys. Rev. Lett. 97 (2006) 262002.

[23] S. Morrow et al., Eur. Phys. J. A 39 (2009) 5.

[24] A.V. Radyushkin, Phys. Rev. D 59 (1999) 014030; Phys. Lett. B 449 (1999) 81.

[25] C. Hadjidakis et al., Phys. Lett. B 605 (2005) 256.

[26] D.G. Cassel et al., Phys. Rev. D 24 (1981) 2787.

[27] A. Airapetian et al., Eur. Phys. J. C 17 (2000) 389.

[28] M. R. Adams et al., Z. Phys. C 74 (1997) 237. 